\title{
3D BEM-based cooling-channel shape optimization for injection molding processes
}

\author{
N. Pirc ${ }^{1,2, a}$, F. Schmidt ${ }^{1}$, M. Mongeau ${ }^{2}$ and F. Bugarin ${ }^{2}$ \\ 1 CROMeP - École des Mines d'Albi-Carmaux, Campus Jarlard, 81013 Albi, Cedex 9, France \\ 2 Université de Toulouse, LAAS-CNRS, and Institut de Mathématiques, UPS 31062 Toulouse Cedex 9, France
}

Received 30 March 2008, Accepted 15 Juin 2008

\begin{abstract}
Today, around $30 \%$ of manufactured plastic goods rely on injection molding. The cooling time can represents more than $70 \%$ of the injection cycle. Moreover, in order to avoid defects in the manufactured plastic parts, the temperature in the mold must be homogeneous. We propose in this paper a practical methodology to optimize both the position and the shape of the cooling channels in 3D injection molding processes. For the evaluation of the temperature required both by the objective and the constraint functions, we must solve 3D heat-transfer problems via numerical simulation. We solve the heat-transfer problem using Boundary Element Method (BEM). This yields a reduction of the dimension of the computational space from 3D to 2D, avoiding full 3D remeshing: only the surface of the cooling channels needs to be remeshed at each evaluation required by the optimization algorithm. We propose a general optimization model that attempts at minimizing the desired overall low temperature of the plastic-part surface subject to constraints imposing homogeneity of the temperature. Encouraging preliminary results on two semi-industrial plastic parts show that our optimization methodology is viable.
\end{abstract}

Key words: BEM; injection molding; SQP; cooling channel optimization

\section{Introduction}

Today, around $30 \%$ of manufactured plastic goods rely on injection molding, which is based on the injection of a fluid plastic material into a closed mold (Fig. 1) (Madehow [1]) displays an illustrative injection molding process). The cooling time can represent more than $70 \%$ of the injection cycle. Moreover, in order to avoid defects in the manufactured plastic parts, the temperature in the mold must be homogeneous. Thus, the design and the position of the cooling channels are crucial elements in the design of the mold. In order to decide the position and the shape of the cooling channels in the mold, designers commonly rely on experience and intuition within a costly trial-and-error design process. This manual design process becomes inadequate and unpractical for complex problems. This is particularly true nowadays with rapid prototyping processes such as layered design or selective laser sintering that enable manufacturers to build almost any desired shape of cooling channel geometry in the mold. As a consequence, designers need a more powerful tool integrating the cooling analysis, its numerical simulation, and even optimization algorithms into the design process.

We propose in this paper a practical methodology to optimize both the position and the shape of the

\footnotetext{
${ }^{a}$ Corresponding author: pircpirc@enstimac.fr
}

cooling channels in 3D injection molding processes. For the evaluation of the temperature, required both by the objective and the constraint functions, we must solve 3D heat-transfer problems via numerical simulation. Severe numerical methods such as Finite Element Method (FEM) [2] or Boundary Element Method (BEM) [3] can be used for solving the heat-transfer problem. Silva [4] used 3D FEM software to model the injection molding cycle for complex geometries of molds. However, the computational burden of 3D FEM makes the integration of an optimization algorithm unpractical. Indeed, a FEM approach to the 3D heat-transfer problem imposes severe storage and $\mathrm{CPU}$ requirements, even if moderately complex industrial parts were to be targeted. An optimization algorithm requires numerous (and here expensive in CPU time) objective-function evaluations. Moreover, unless one knows a very good initial design, from one optimization iteration to the next the design is generally significally different. As a consequence, the capability of FEM to handle (small) shape perturbations for 3D mesh cannot be advantageously exploited here. BEM is a method that was popularized by Brebbia [5]. It is used in many applications, such as gas-assisted injection molding processes [6], or groundwater flow and mass transport problems [7]. BEM transforms domain integrals into boundary element integrals, involving therefore a discretization restricted only to the external and internal 


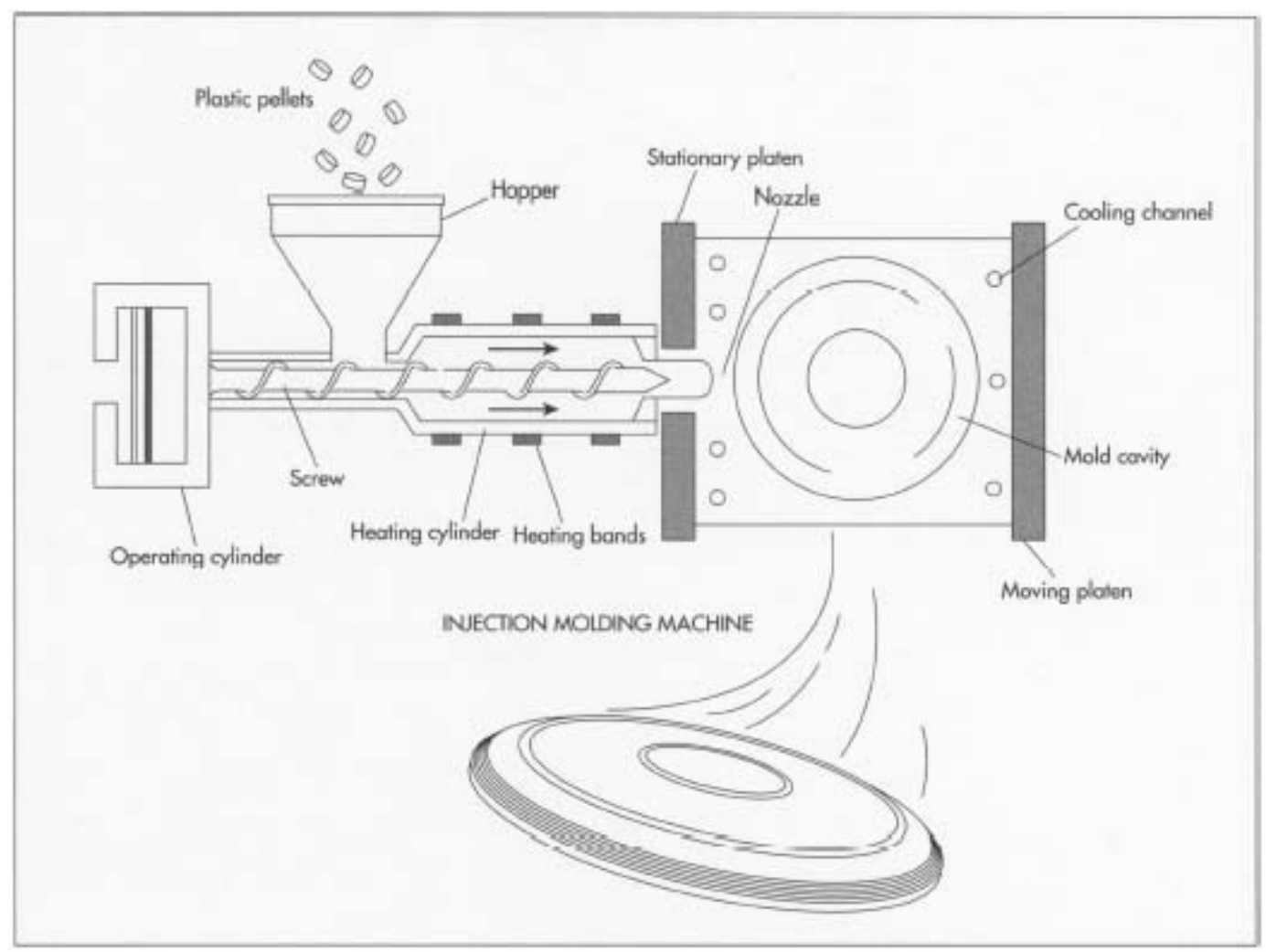

Fig. 1. Injection unit.

field boundary. An optimization method can therefore be envisaged to modify the position and also the shape parameters of the cooling channels in order to improve the cooling performance of the mold.

Park [8] proposed such a BEM-based procedure for the position of the cooling channels relying on augmentedLagrangian optimization method. However, his study is restricted to molds that are generalized cylinders, amenable to 2D molds. His optimization model minimizes the variations of the temperature distribution on the cavity surface with respect to the average temperature. As a consequence, his optimal configuration provides a uniform but high average temperature, leading a very long cooling time which is not desirable in the context of large-scale manufacturing. Mathey [9] also used BEM to solve the heat-transfer problems with a Sequential Quadratic Programming (SQP) [10] algorithm to improve mold injection cooling. She minimizes an objective function which is the weighted sum of two criteria. Her first criterion is the average temperature at the plastic-part surface. Her second criterion is the sum of the temperature variations with respect to the average temperature. Moreover, Mathey optimizes both the position and the shape of the cooling channels. However, her approach is also restricted to 2D molds (as for Parks [8]), BEM then reduces the dimension of the computation space from $2 \mathrm{D}$ to $1 \mathrm{D})$.

Our contribution is threefold. First, we address 3D mold geometries with a BEM approach reducing the dimension of the computation space from $3 \mathrm{D}$ to $2 \mathrm{D}$, avoiding full 3D remeshing: only the surface of the cool- ing channels needs to be re-meshed at each evaluation required by the optimization algorithm. Secondly, we propose a general optimization models that attempts at minimizing the desired overall low temperature of the plasticpart surface subject to constraints imposing homogeneity of the temperature. Thirdly, we demonstrate that our optimization methodology is viable with encouraging preliminary results on two semi-industrial plastic parts. The paper is organized as follows. We detail in the next section the 3D heat-transfer problem that we must solve for every optimization evaluation of the temperature required by the optimization algorithm. Section 3 describes our overall optimization methodology. In order to validate our approach, we report encouraging preliminary results in Section 4. We conclude in Section 5.

\section{The heat transfer problem}

This section describes the heat-transfer problem that must be solved at every temperature evaluation required by the optimization algorithm:

As shown in Figure 2, after a few cycles the variation of the temperature in the injection mold in production can be considered to be quasi-stationary [11]. Once the average temperature of the mold is stabilized, the cycle-averaged approach can predict well the overall performance of the cooling system. Thus, we can consider a stationary regime neglecting the transitory oscillations of the temperature. For a metallic mold, when between $0{ }^{\circ} \mathrm{C}$ 


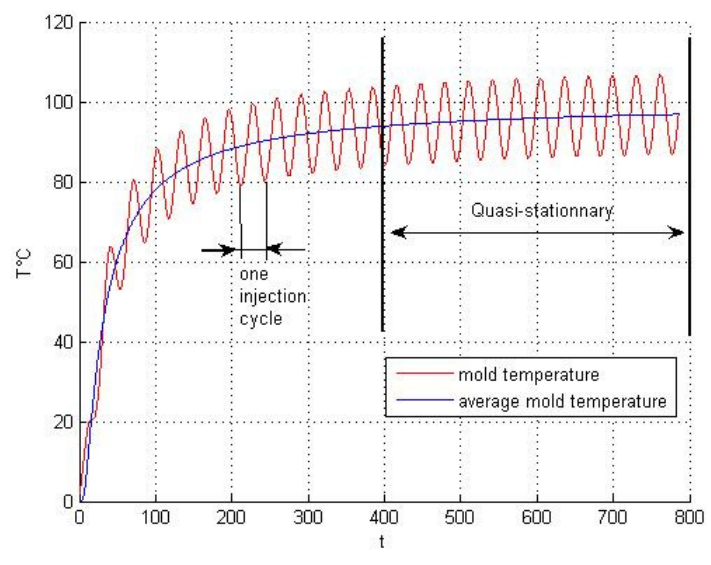

Fig. 2. Temperature at the surface of the mold versus time (in seconds).

to $200{ }^{\circ} \mathrm{C}$, the thermal conductivity can be considered as a constant. Thus, the stationary heat conduction problem reduces to the following Laplace equation:

$$
\Delta T=0,
$$

where $\Delta$ is the Laplacian operator, $T$ is a temperature vector. Multiplying this equation by a weighting function $T^{*}$, and using Green's theorem, we obtain the well-known Somigliana's equation [5]. The following Green integral representation formula gives the value of $T$ in terms of integral equations involving the fundamental solution $T^{*}$ of the Laplace equation:

$$
C \cdot T+\int_{\Gamma} T \cdot\left(\nabla T^{*} \cdot n\right) \cdot d \Gamma=\int_{\Gamma}(\nabla T \cdot n) \cdot T^{*} \cdot d \Gamma .
$$

Here, $n$ is the unit normal at one element, $\Gamma$ is the boundary of the domain, $\mathrm{C}$ is equal to 1 inside the domain $\Omega$ and to 0.5 on its boundary $\Gamma$. The weighting function $T^{*}$ and the flux $q^{*}$ are the fundamental solutions of the heat-problem, the so-called Green's functions [5]:

$$
T^{*}=\frac{-1}{2 \cdot \pi \cdot r} \text { and } q^{*}=\frac{-r \cdot n}{4 \cdot \pi \cdot r^{2}},
$$

where $r$ is the distance from the point of application of the concentrated unit source to any other point under consideration. Note that in equation (1) all the integrals are taken over the boundary of the domain. For the computation of the heat-transfer problem, the contour $\Gamma$ is discretized and the integrals in the above equation are defined in terms of nodal values by means of interpolation functions. After reorganization of terms in equation (2) using the boundary conditions, we obtain a nonsymmetrical linear system. We shall need to remesh the cooling channel surfaces using 2D elements, and to solve this system of equations at every optimization evaluation.

Figure 3 shows the boundary conditions on the mold. We detail the boundary conditions of the heat transfer problem in Figure 3. The following equation relates the

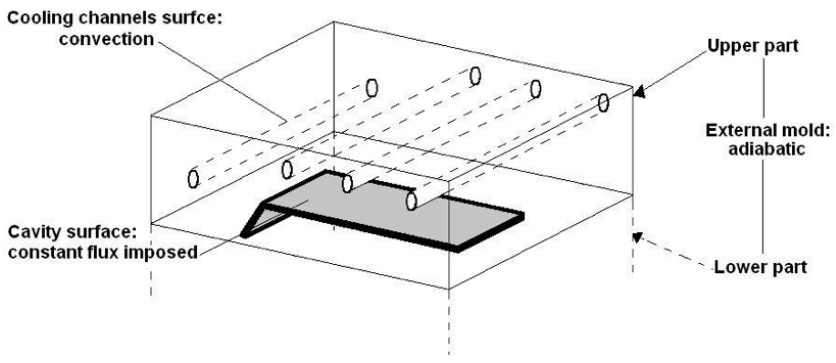

Fig. 3. Boundary conditions.

Table 1. Parameter values for heat-transfer problem (from [9])

\begin{tabular}{clcc}
\hline & Units & Polymer (PP) & Mold (steel 40cmd8s) \\
\hline$\lambda$ & $\mathrm{W} \mathrm{m}^{-1} \mathrm{~K}^{-1}$ & 0.63 & 34 \\
$\rho$ & $\mathrm{kg} \mathrm{m}^{-1}$ & 891 & 7800 \\
$C_{p}$ & $\mathrm{~J} \mathrm{~kg}^{-1} \mathrm{~K}^{-1}$ & 2740 & 460 \\
\hline
\end{tabular}

temperature of the coolant $T_{c}$, and the heat-transfer coefficient $h$, with the coolant flow rate (via Colburn correlation coefficient) [12]:

$$
\lambda . q=h\left(T_{a}-T_{c}\right)
$$

where $\lambda$ is the conductivity, $q$ is the heat flux, and $T_{a}$ is the ambient temperature. The following polymer properties are referenced in Table 1: $\rho$ is the density and $C_{p}$ is the heat capacity. The flux density at the cavity surface, $\phi_{\text {cavity }}$, is computed from the cooling cycle time, $t_{\text {cooling }}$, and the polymer properties (heat due to polymer crystallization is neglected) [12]:

$$
\phi_{\text {cavity }}=\frac{Q}{t_{\text {cooling }} \cdot \Gamma_{\text {cavity }}},
$$

where $Q$ is the heat evacuated by the plastic part during one injection cycle and $\Gamma_{\text {cavity }}$ is the area of the cavity.

Finally, remark that for our problem, we only need to mesh once the cavity surface and the external surface of the mold. Then, each time the optimization algorithm requires to evaluate the objective and the constraint functions, we must mesh the surface of the cooling channels. The output of the heat-transfer problem resolution is a set of temperature measurements, one for each surface element: cavity, external mold surface, and cooling channels. However, we only need the vector $T$ of temperature measurements, $\left\{T_{i}(x)\right\}_{i \in S}$, at the cavity surface elements ( $S$ denotes the index set of the cavity surface elements).

\section{Overall optimization methodology}

We first present in this section how we formulate our problem under a mathematical programming form. Then, we detail our overall computational methodology.

In the sequel, $x$ will denote the vector of optimization variables (position and shape parameters for the cooling 


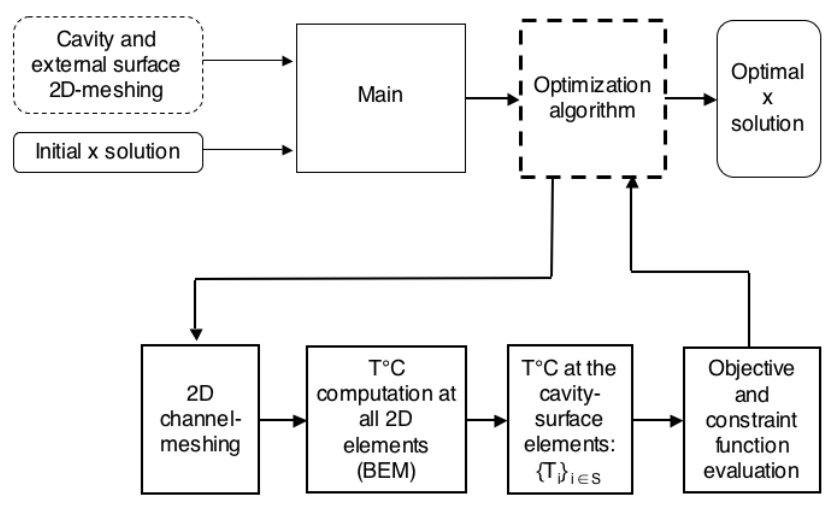

Fig. 4. The heat-transfer simulation coupled with the optimization algorithm.

channels). Since the output of the heat-transfer problem is function of $x$, we shall make explicit the dependence of the temperature measurements upon the position and shape parameters $x:\left\{T_{i}(x)\right\}_{i \in S}$.

Most practical optimization problems involve several (often contradictory) objective functions. It is the case here as one aims at minimizing the temperature of the plastic-part surface while minimizing the variation of the temperature along this surface. The simplest way to proceed in such a multicriterion context is to consider as objective function a weighted sum of the various criteria, which involves choosing appropriate weighting parameter values (more on multicriterion optimization can be found in [13]). An obvious alternative is to use one criterion as objective function while requiring in constraints maximal threshold levels for the remaining criteria. We choose here the latter approach because in our application context, it is more desirable to consider the temperature requirement as a constraint. Indeed, we do know a threshold level value for the maximal temperature variation under which any variation is equally acceptable. More precisely, we formulate our problem under the form:

$$
\begin{array}{ll}
\min _{x}\|T(x)\| & \\
\text { subject to } & f(T(x)) \leq 0 \\
& g(x) \leq 0,
\end{array}
$$

where $f$ is a real-valued function used to stipulate the uniformity-temperature constraint, and $g(x)$ is a general vector-valued non-linear function. Remark that $\|\cdot\|$ stands for any norm such as the $l_{1}$ norm (sum of absolute values), the standard Euclidean $\left(l_{2}\right)$ norm, or the $\max \left(l_{\infty}\right)$ norm. The general constraints $(4)$ represent any geometry-related or other industrial constraints, such as:

- upper/lower-bound constraints on the $x_{i}$ 's;

- keeping the cooling channels within the mold;

- technically-forbidden zones where we cannot position the cooling channels (for instance due to the presence of ejectors);

- constraints stipulating a minimal distance between every pair of cooling channels to avoid inter-channels collision.

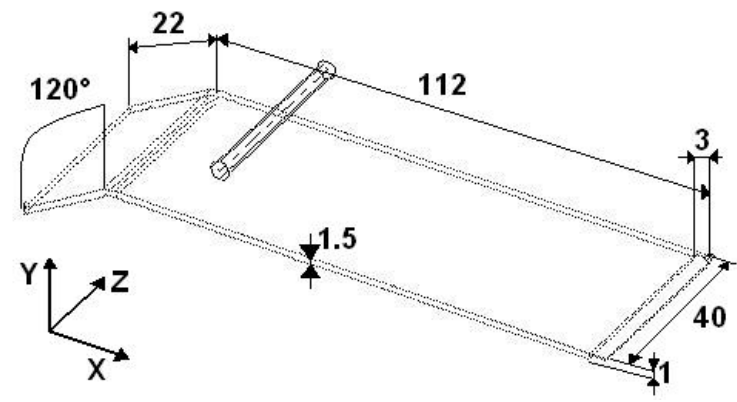

Fig. 5. Plastic part dimensions and one cooling channel.

Figure 4 shows the coupling between the thermal solver and the optimization algorithm.

\section{Applications}

In this section, we present computational experiments on two semi-industrial plastic parts.

For both applications, we specify the following implementation strategies. We use commercial software for the tasks represented by dashed-line boxes in Figure 4:

- IDEAS for the initial 2D meshing of the cavity and the external surface (note that this meshing remains constant throughout the optimization process);

- Matlab's fmincon Sequential Quadratic Programming subroutine [10] for the optimization algorithm (with finite-difference computation of the gradients for these preliminary experiments - in future work, we intend to compute exact gradients).

We programmed every other task (represented by full-line boxes in Fig. 4) in Matlab. The system of linear equations involved in the heat-transfer problem is solved by the LApack subroutine included in Matlab 7.0 [14]. We use here the $l_{\infty}(\max )$ norm for the objective function:

$$
\|T(x)\|_{\infty}:=\max _{i \in S} T_{i}(x) .
$$

All numerical results reported in the sequel are obtained on a Macintosh $1.83 \mathrm{GHz}$ Intel core 2 duo.

\subsection{EuroTooling mold with straight cooling channels}

For our first application, we consider a semiindustrial injection mold design for the European project: EuroTooling 21. The plastic part produced from this mold is a bended plate whose dimensions are shown in Figure 5 (in $10^{-3} \mathrm{~m}$ ). We want to optimize the position of the cooling channels. The channels are simple straight horizontal cylinders of constant length as illustrated in Figure 6 . In order to simplify the presentation, in this application we only optimize the position of the cylinders (we could also consider optimizing the radius of the cross-section 


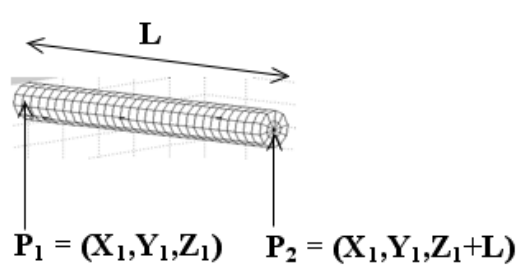

(a)

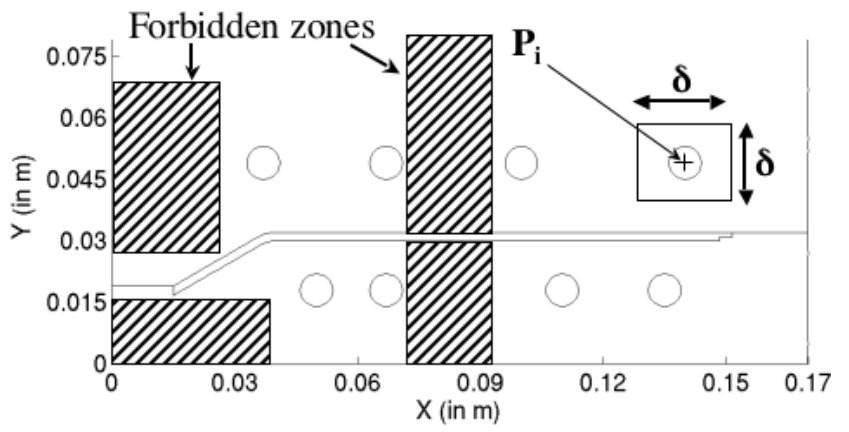

(b)

Fig. 6. Cooling channel parameters.

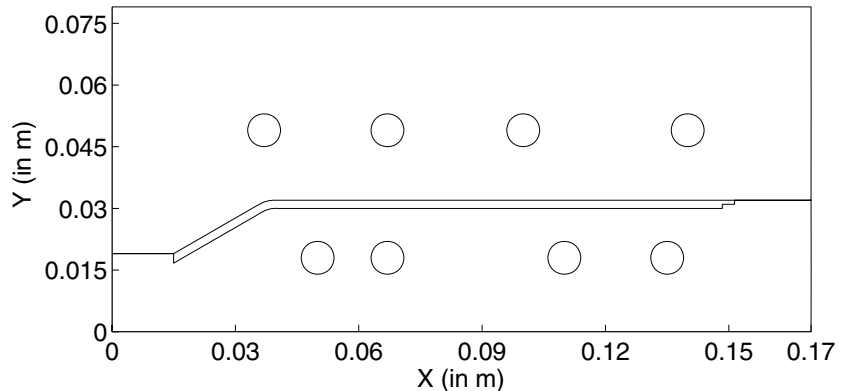

Fig. 7. Initial and optimized (dashed lined) positions of the cooling channels.

of the channels and the number of channels, etc.). As a consequence, three parameters suffice to describe the position of each cooling channel. For illustration purposes we consider here 8 cooling channels. The components of the optimization vector $x$ are here the coordinates of each end point $\left(X_{i}, Y_{i}\right), i=1 \ldots 8$. (in our application $Z_{i}$ is fixed). Thus, our problem involves 16 optimization variables. For this application, we use the constraint function $F(T(x))=\|T(x)-\bar{T}(x)\|_{\infty}-\sigma$, where the difference $T(x)-\bar{T}(x)$ stands here for the vector whose $i$ th component is $T_{i}(x)-\bar{T}(x), \bar{T}(x):=\frac{1}{|S|} \sum_{i \in S} T_{i}(x)$ is the average of the $T_{i}$ 's, and $\sigma$ is a user-defined temperature uniformity tolerance. We choose here $\sigma=4{ }^{\circ} \mathrm{C}$. In other words, we do not accept variations of the temperature that are above $4{ }^{\circ} \mathrm{C}$ with respect to the average temperature and this, everywhere on the plastic-part surface. The remaining constraints here simply stipulate lower/upper bounds and also forbid the grey zones displayed in Figure 6.

The 2D meshing of the mold surfaces is displayed in Figure 10. The surface of each channel is discretized into 320 quadrangles. We use as starting point, a heuristic solution provided by an experienced engineer. On average, one objective-function evaluation (i.e. the computation of the temperatures) requires $86 \mathrm{~s}$ of CPU time. Since for these preliminary experiments we are content with computing gradients using finite-difference approximations, one optimization iteration involves 26 min of CPU time

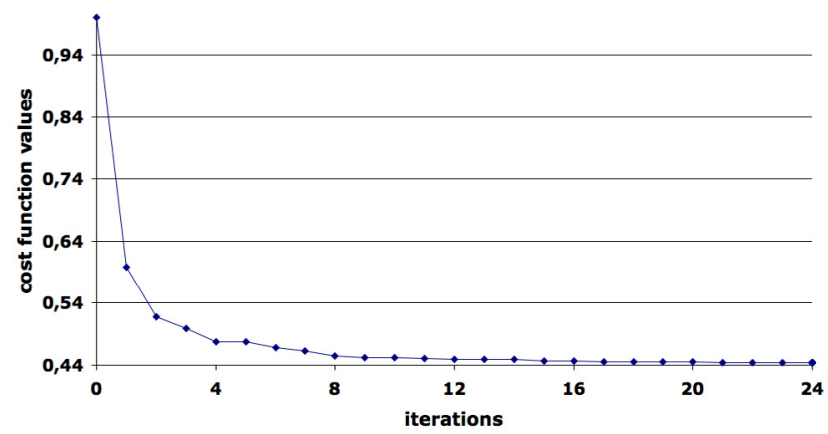

Fig. 8. Objective function versus iterations.

on average. Figure 7 displays initial (full lines) and optimal (dashed lines) positions of the cooling channels. Figure 8 displays the decrease of the objective-function value in terms of the number of optimization iterations. We observe in Figure 8 that the objective function is reduced by $90 \%$ of its initial value within the first four optimization iterations.

Figure 9 shows the temperature distribution along the surface of the cavity mold before and after optimization (points $A, B$ and $B^{\prime}$ refer to positions in Fig. 7). We observe that both the temperature variance and the average temperature decreased significantly. The temperature distribution after optimization can be seen in Figure 10

\subsection{Plastic-cup mold with a helix cooling channel}

We now report computational results on a 3D plastic part: a plastic cup. The height of the mold and that of the plastic cup are respectively 0.12 and $0.08 \mathrm{~m}$. The upper radius of the cup is $0.06 \mathrm{~m}$, its lower radius is $0.04 \mathrm{~m}$, and its thickness is $3 \times 10^{-4} \mathrm{~m}$. In this preliminary study, we are content with considering a helix-shape cooling channel with circular cross section, with only three degrees of freedom: the section diameter of the cooling channel, the frequency of the turn and its overall diameter. The cooling channel is therefore defined in $\Re^{3}$ using the single 


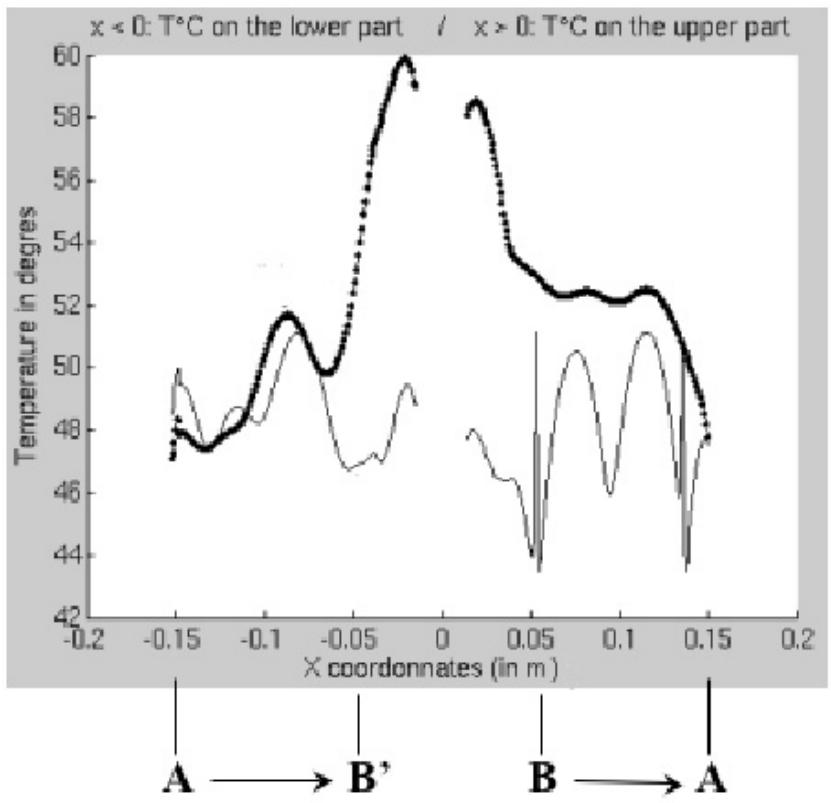

Fig. 9. Temperature measurements along the surface of the mold cavity before (black) and after (grey) optimization.

parameter algebraic equation:

$$
\begin{aligned}
& X(t)=r_{s} \cos t \\
& Y(t)=r_{s} \sin t \\
& Z(t)=t,
\end{aligned}
$$

where $r_{s}$ is the radius of the spiral. We discretize this curve into 63 straight-cylinder channels (Fig. 12) with radius $r_{c}$. Each of these cylinders is further discretized into 20 quadrangle elements as shown in Figure 13. The 2D meshing of the mold surfaces is displayed in Figure 11. The optimization variables here are:

- the geometrical parameters that control the shape of the helix, that is to say: the radius, $r_{s}$ of the spiral and the number, $n$, of helix turns;

- the radius $r_{c}$ of the circular cross section of the cylinder channels.

Our temperature homogeneity constraint here is:

$$
\max _{i \in S} T_{i}(x)-\min _{i \in S} T_{i}(x) \leq \sigma,
$$

with $\sigma=4{ }^{\circ} \mathrm{C}$. The meshing of the mold surfaces is shown in Figure 12. On average, one objective-function evaluation (temperature computation) requires $82 \mathrm{~s}$ of $\mathrm{CPU}$ time. Again, because of the fact that we compute gradients using finite-difference approximation, one optimization iteration involves, on average, $7 \mathrm{~min}$ of CPU time. Figure 14 shows the helix curve before and after optimization. Figure 15 displays the decrease of the objectivefunction value in terms of the number of optimization iterations. We observe a rapid convergence during the first five iterations. Table 2 displays the initial and optimal solutions, and Figure 16 shows the temperature distribution at the surface of the mold after optimization.

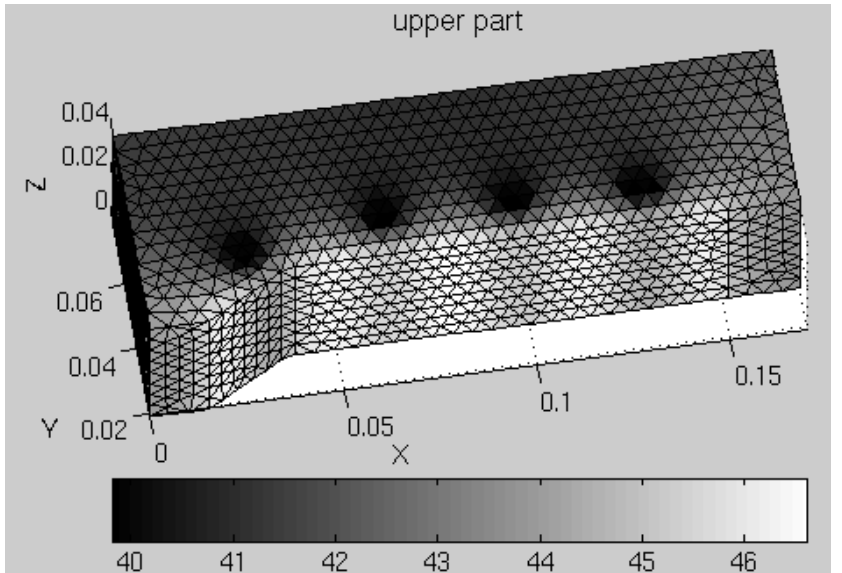

(a)

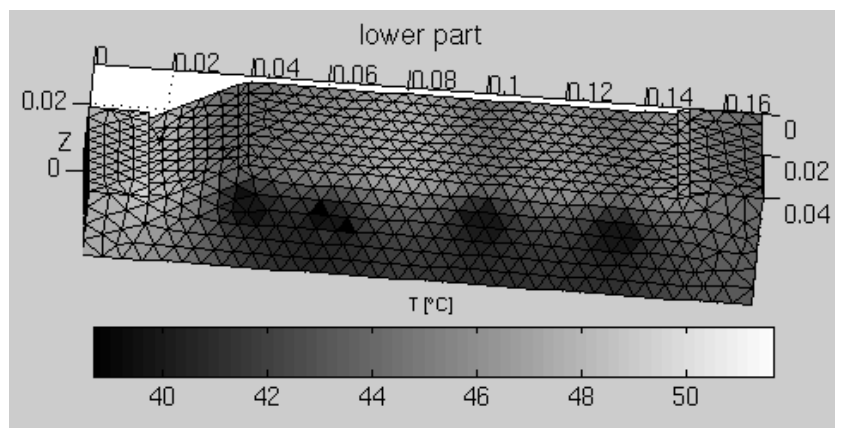

(b)

Fig. 10. Temperature at the surface of the $3 \mathrm{D}$ mold after optimization.

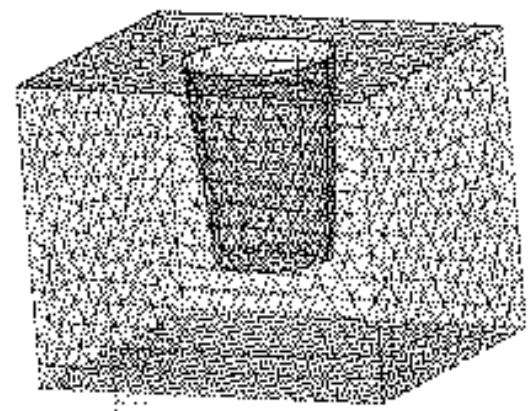

Fig. 11. Meshing of the surfaces for the plastic-cup mold.

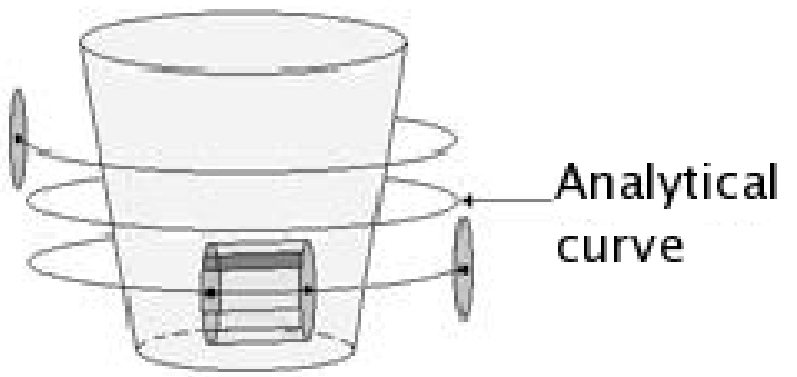

Fig. 12. Discretization into straight cylinder channels. 


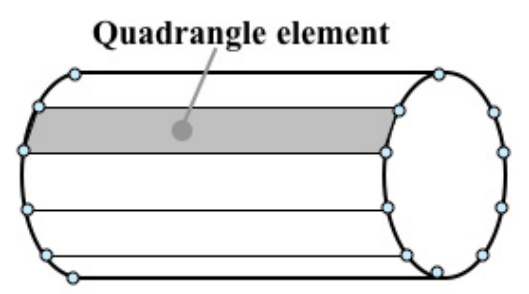

Fig. 13. Cooling-channel surface meshing.

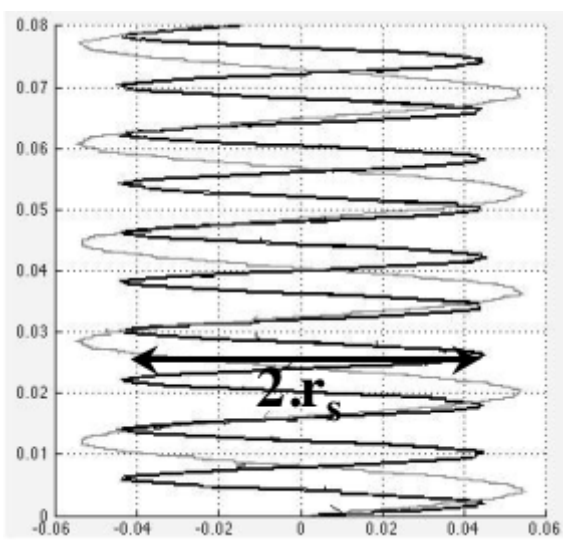

Fig. 14. Helix curve before (black) and after (grey) optimization.

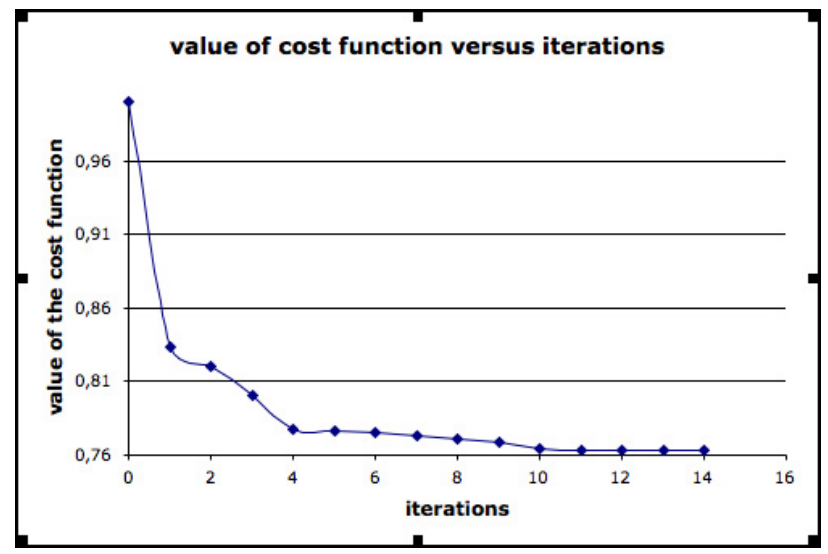

Fig. 15. Objective-function value versus iterations.

Table 2. initial and optimal solutions.

\begin{tabular}{ccc}
\hline & $\begin{array}{c}\text { Before } \\
\text { optimization }\end{array}$ & $\begin{array}{c}\text { After } \\
\text { optimization }\end{array}$ \\
\hline$r_{s}(\mathrm{~m})$ & 0.045 & 0.055 \\
$r_{c}(\mathrm{~m})$ & 0.003 & 0.001 \\
$n$ & 10 & 5 \\
objective-function value & $35{ }^{\circ} \mathrm{C}$ & $28^{\circ} \mathrm{C}$ \\
$\max T_{i}-\min T_{i}$ & $5{ }^{\circ} \mathrm{C}$ & $2{ }^{\circ} \mathrm{C}$ \\
\hline
\end{tabular}

\section{Conclusion}

We proposed in this paper a practical methodology to optimize both the position and the shape of the cooling channels in industrial 3D molds. This was made possible through the use of the boundary elements method that avoids full 3D remeshing. Indeed, only the surface

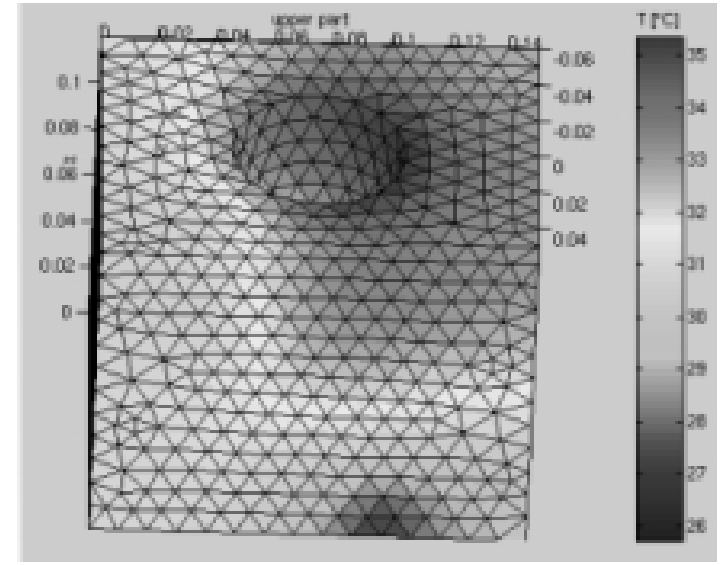

(a)

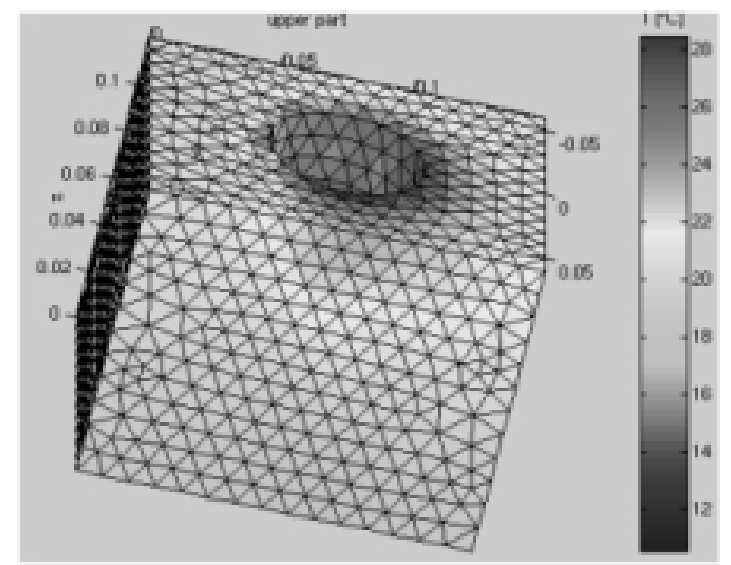

(b)

Fig. 16. Temperature at the surface of the $3 \mathrm{D}$ mold after optimization.

of the cooling channels needs to be remeshed to solve the heat-transfer problem involved each time the optimization algorithm needs to evaluate the temperature at the plastic part surface. Our optimization model can account for various ways to address the overall desired lowtemperature criterion and the temperature-homogeneity constraint. Encouraging computational experiments on two semi-industrial plastic-parts, demonstrated the viability of our approach that is intended to be used as a decision-analysis tool for designing new, original mold geometries.

We are currently implementing exact gradients to replace finite-difference approximations in order to improve the efficiency of the optimization. Future work could allow more complex shape variations. Recall that we considered in this preliminary study only three degrees of freedom in order to test the viability of our optimization methodology. Other degrees of freedom for our plasticcup application could for instance include the flux in the cooling channel and a vertical angle so that the helix runs along a cone (closer to the actual cup shape). Finally, further work could formulate our design problem as a topology optimization problem in order to offer even 
larger design freedom. The wide range of geometries we can tackle for the cooling channels will bring us to address more complex industrial molds. This will undoubtly yield challenging global optimization problems, as this can be anticipated based on the numerical experiments we reported $[15,16]$.

\section{Acknowledgements}

This study was conducted within the framework of the European project EuroTooling 21 (IP 505901-5), www. eurotooling21.com.

\section{References}

1. www.madehow.com/Volume-5/Frisbee.html

2. S. Kolossov, E. Boillat, R. Glardon, P. Fischer, Internat. J. Machine Tools 44, 117 (2004)

3. A. Polynkin, Intern. Polymer Process. XIX, 108 (2004)

4. L. Silva, Intern. Polymer Process. XX, 265 (2005)

5. C. Brebbia, J. Domiguez, Boundary elements: An introductory course, WIT Press/Computational Mechanics Publication, 1992
6. K.E. Khayat, J. Non-Newtonian Fluid Mechanics 57, 253 (1994)

7. K.L. Katsifarakis, Engineering Anal. Boundary elements 23, 555 (1999)

8. S.J. Park, Intern. J. Numer. Methods Eng. 43, 1109 (1998)

9. E. Mathey, Automatic optimization of the cooling of injection mold based on the boundary element method. NumiForm 2004 Proceedings (2004) pp. 222-227

10. J. Nocedal, S.J. Wright, Numerical optimization. Springer, 2nd ed., 2006

11. I. Catic, A. Abadzic, M. Rujnic-Sokele, J. Injection Molding Technology 3, 194 (1999)

12. L. Jui-Ming, J. Injection Molding Technology 6, (2002)

13. M. Ehrgott, Multicriteria Optimization Springer 2nd ed., 2005

14. LApack, www.netlib.org/lapack/

15. A. Carlos, D. Alan, Engineering Optim., 31, 337 (1999)

16. H.J. Lee, Y.D. Kim, Engineering Optimization 40, 17 (2008)

17. The MathWorks, User's Guide. Optimization Toolbox For Use With Matlab, Version 2

18. Y. Wen-Hsien, Three-dimensional simulation of injectioncompression molding of a compact disc. ANTEC 2001 Conference Proceedings (2001) 